\title{
Differential Membrane Protein Profile in Bovine X- and Y-Sperm
}

Dan Shen ${ }^{1 \dagger}$, Chenghao Zhou ${ }^{1 \dagger}$, Mingyue Cao ${ }^{1}$, Wentao Cai ${ }^{1}$, Hongwei Yin $^{1}$, Li

Jiang $^{1^{*}}$, and Shengli Zhang ${ }^{1^{*}}$

1Key Laboratory of Animal Genetics, Breeding and Reproduction, Ministry of

Agriculture \& National Engineering Laboratory for Animal Breeding, College of

Animal Science and Technology, China Agricultural University, Beijing 100193,

China

*CORRESPONDENCE:

Shengli Zhang

zhangslcau@cau.edu.cn

Li Jiang

lijiang@cau.edu.cn

$\dagger$ Equal contribution.

AUTHOR INFORMATION

\section{Corresponding Authors}

Dan Shen - Key Laboratory of Animal Genetics, Breeding and Reproduction, Ministry of Agriculture \& National Engineering Laboratory for Animal Breeding, 
College of Animal Science and Technology, China Agricultural University, Beijing 100193, China; orcid.org/0000-0002-2231-0224; Email: qingchunzhishi@,163.com

Shengli Zhang - Key Laboratory of Animal Genetics, Breeding and Reproduction, Ministry of Agriculture \& National Engineering Laboratory for Animal Breeding, College of Animal Science and Technology, China Agricultural University, Beijing 100193, China; orcid.org/0000-0002-9780-515X; Email: zhangslcau@cau.edu.cn

Li Jiang - Key Laboratory of Animal Genetics, Breeding and Reproduction, Ministry of Agriculture \& National Engineering Laboratory for Animal Breeding, College of Animal Science and Technology, China Agricultural University, Beijing 100193, China; Email: lijiang@cau.edu.cn

Chenghao Zhou - Key Laboratory of Animal Genetics, Breeding and Reproduction, Ministry of Agriculture \& National Engineering Laboratory for Animal Breeding, College of Animal Science and Technology, China Agricultural University, Beijing 100193, China; orcid.org/0000-0002-4539-1979; Email: 604242123@qq.com

\section{Author Contributions}

DS and CZ contributed equally to this work. Both SZ and LJ are corresponding authors, with SZ as the first corresponding author. SZ and LJ conceived and designed the study, and revised the manuscript. DS and CZ performed the phenotype collection, sample collection, data analysis, and drafted the manuscript. MC, WC and HY participated in the experimental design and drafted the manuscript. All authors have read and approved the final manuscript.

\section{Notes}


The authors declare no competing financial interest.

\section{Supporting Information}

Mass deviation distribution of all ions (Figure S1); Screening and analysis of proteins used for different research objectives (Figure S2); Protein-protein interaction network of all DEPs (Figure S3); Result of proteins containing predicted transmembrane structures (Figure S4); Venny plot of transmembrane proteins and membrane/extracellular proteins (Figure S5); Raw result of Western blotting of five candidate antigenic proteins and TUBLIN protein (Figure S6); Predicted result of secondary structure and epitopes of $\mathrm{C} 11 \mathrm{H} 2$ orf74 protein (Figure S7) (PDF)

Data of overview of proteins information identified in present study (Table S1); The differentially expressed proteins of X-vs Y-group comparisons (Table S2); List of significant GO terms and KEGG pathways for DEPs detected by GSEA (Table S3); List of proteins predicted to be located in the extracellular or plasma membrane using WoLF PSORT (Table S4); List of proteins predicted to have transmembrane structures and are present on extracellular or plasma membrane (Table S5); Information of the predicted secondary structure and B-cell epitopes of C11H2orf74 (Table S6) (XLSX)

\section{FIGURES}

Figure S1. Mass deviation distribution of all ions. a Measurement of mass accuracy of the peptides. $b$ Measurement of peptide spectrum matching error.

Figure S2. Screening and analysis of proteins used for different research objectives. 
Figure S3. Protein-protein interaction network of all DEPs. Each node represents the differentially expressed protein. Red is the protein with significantly up-regulated abundance or specifically expressed in X-group, while green is the protein with significantly up-regulated abundance or specifically expressed in Y-group. The connection between nodes represents the interaction between proteins, and the thicker the link is, the higher the interaction combine score is, i.e. the higher the credibility of the interaction.

Figure S4. Results of proteins containing predicted transmembrane structures. Each protein expressed in accession in cattle UniProt database, the abscissa represents the length of the amino acid sequence of the protein, and the ordinate represents the probability of localization of different regions on each subcellular component. Red represents transmembrane region, blue represents the inside region, pink represents the outside region.

Figure S5. Venny plot of transmembrane proteins and membrane/extracellular proteins.

Figure S6. Raw results of Western blotting of five candidate antigenic proteins and TUBLIN protein.

Figure S7. Predicted results of secondary structure and epitopes of C11H2orf74 protein. 
Table S1. Overview of proteins information identified in present study.

Table S2. The differentially expressed proteins of X-vs Y-group comparisons.

Table S3. List of significant GO terms and KEGG pathways for DEPs detected by GSEA.

Table S4. List of proteins predicted to be located in the extracellular or plasma membrane using WoLF PSORT.

Table S5. List of proteins predicted to have transmembrane structures and are present on extracellular or plasma membrane.

Table S6. Information of the predicted secondary structure and B-cell epitopes of C11H2orf74. 


\section{Supplementary Figure 1}

a
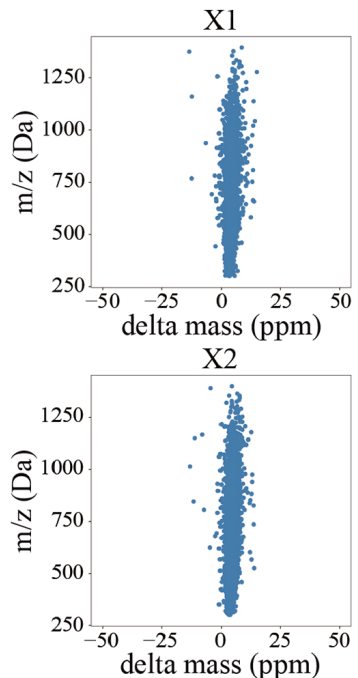

$\mathrm{X} 3$
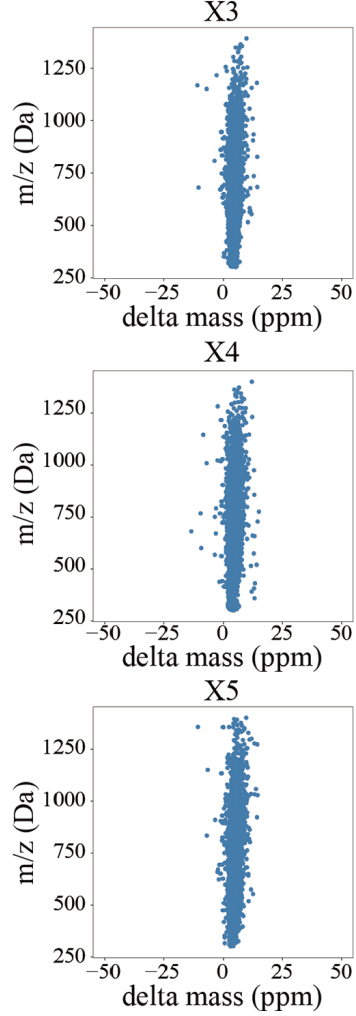
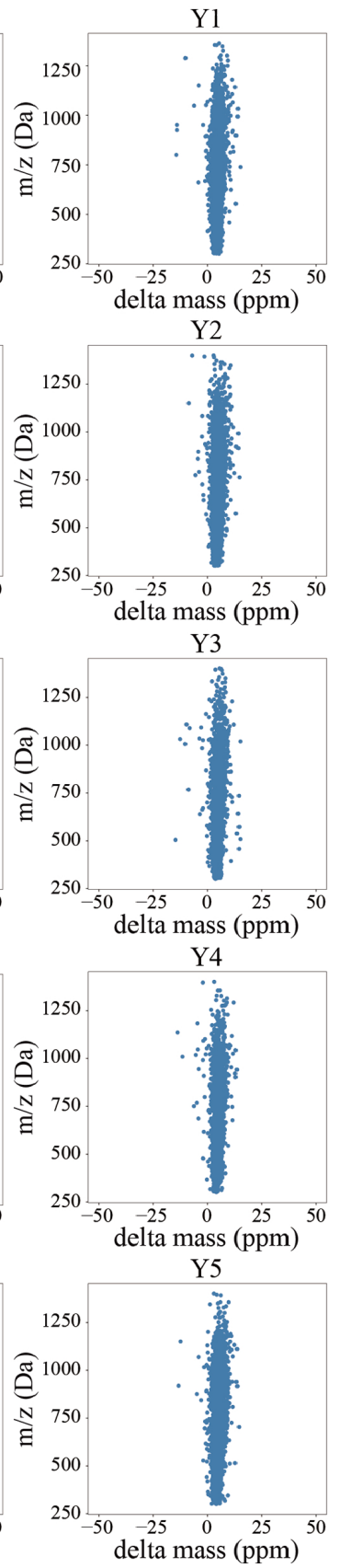

b
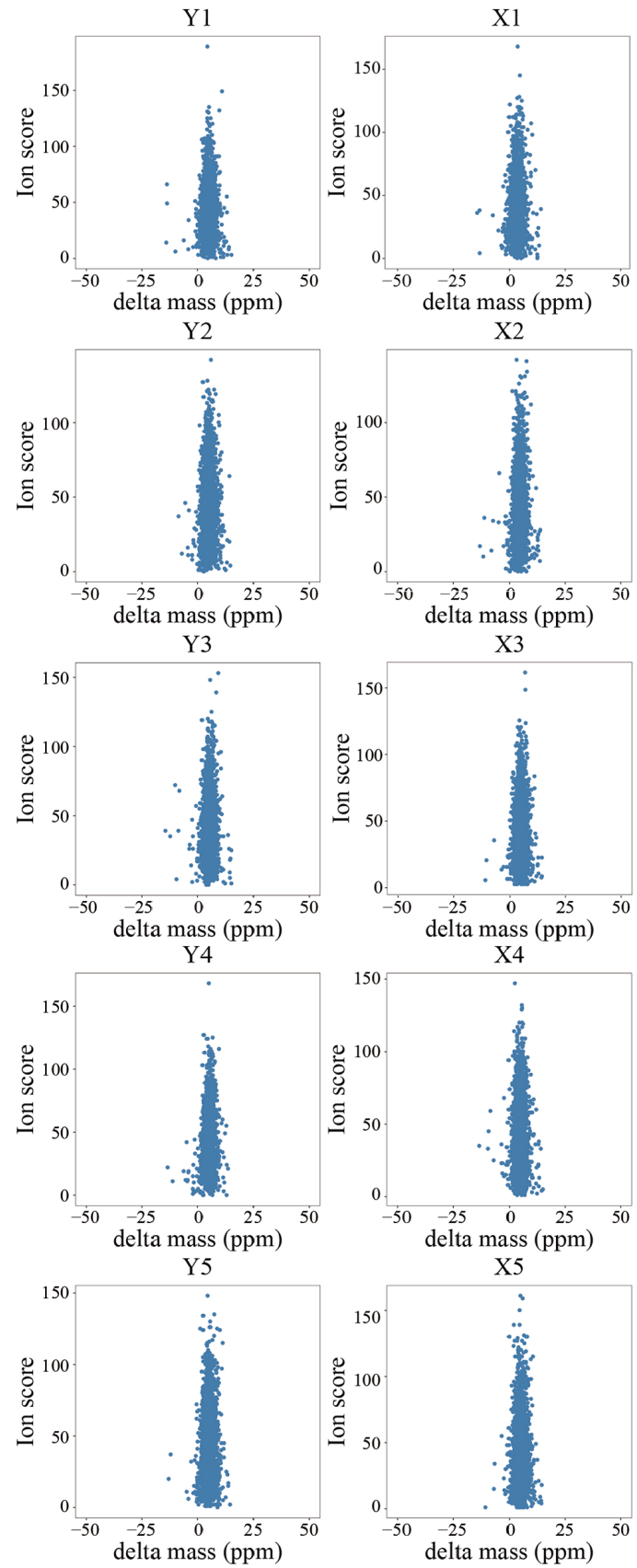


\section{Supplementary Figure 2}

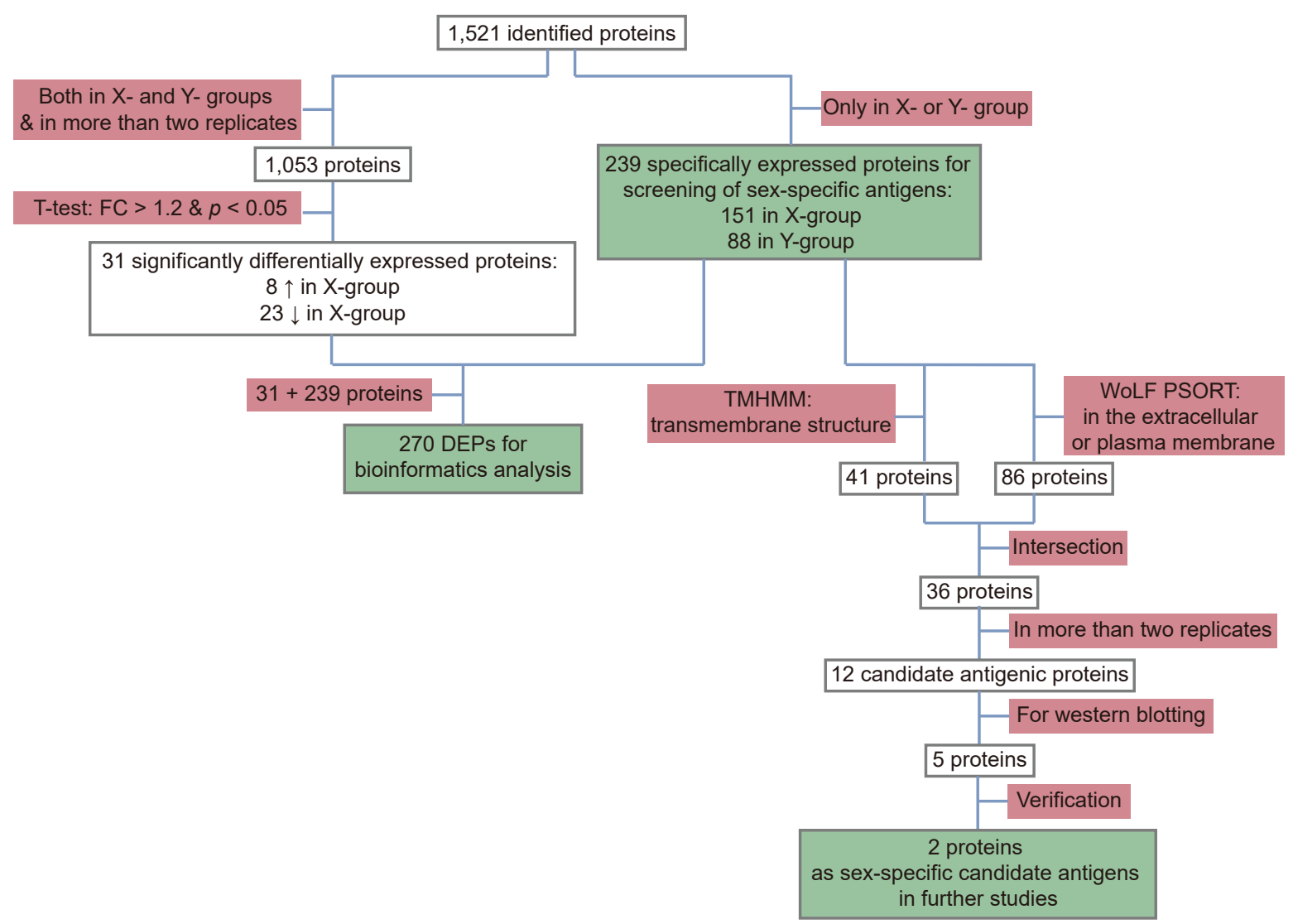




\section{Supplementary Figure 3}

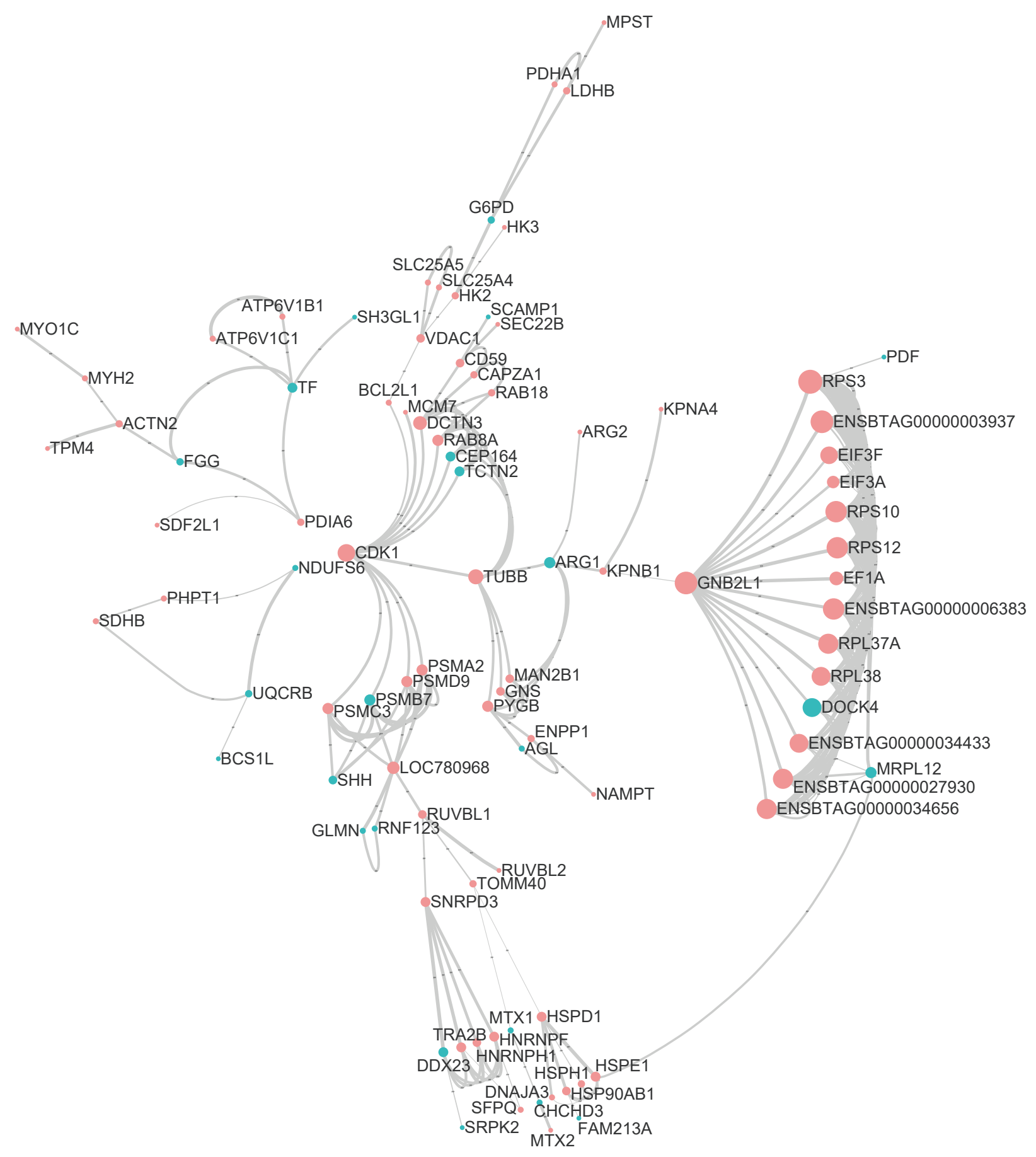




\section{Supplemental Figure 4-1}
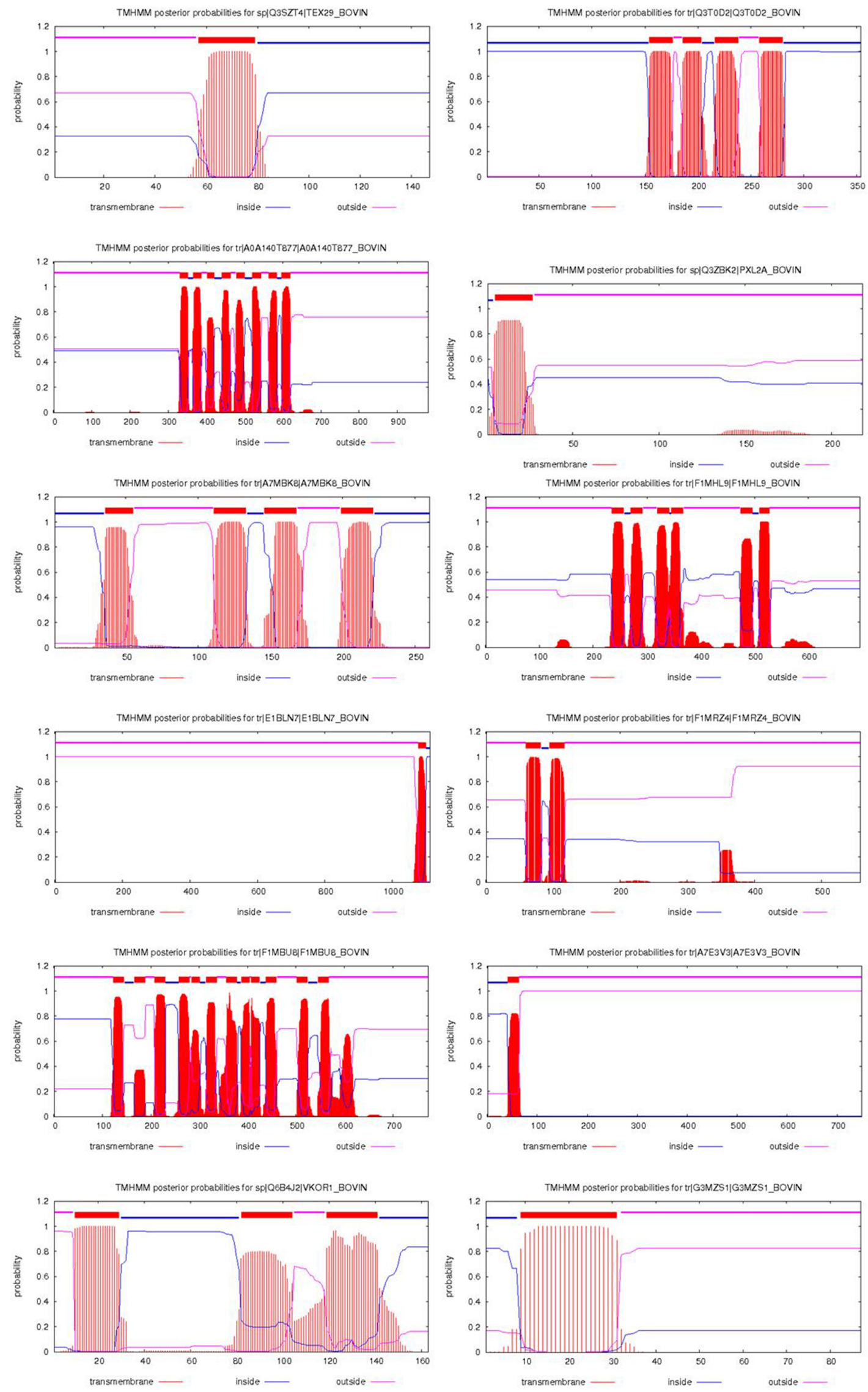


\section{Supplemental Figure 4-2}
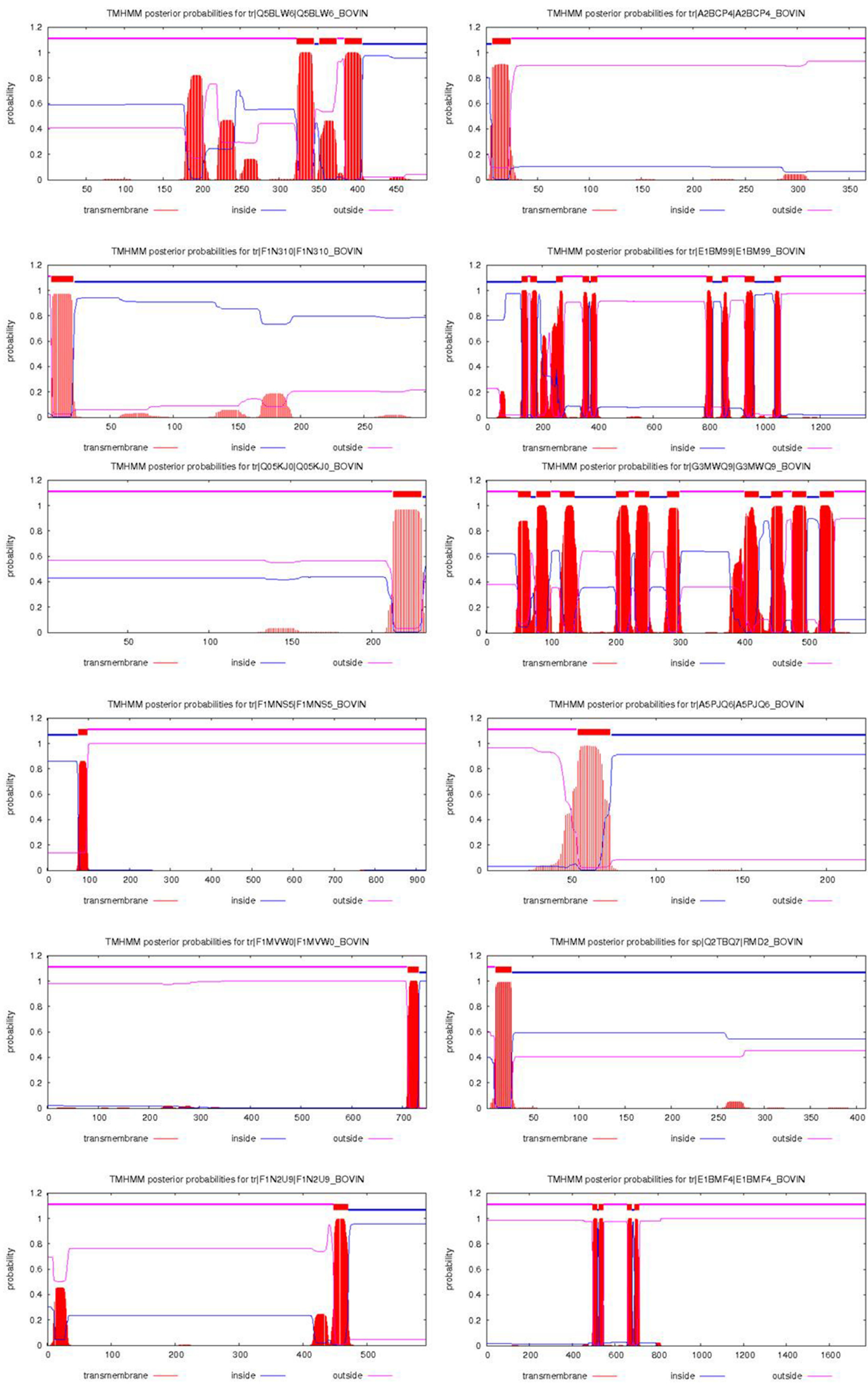


\section{Supplemental Figure 4-3}
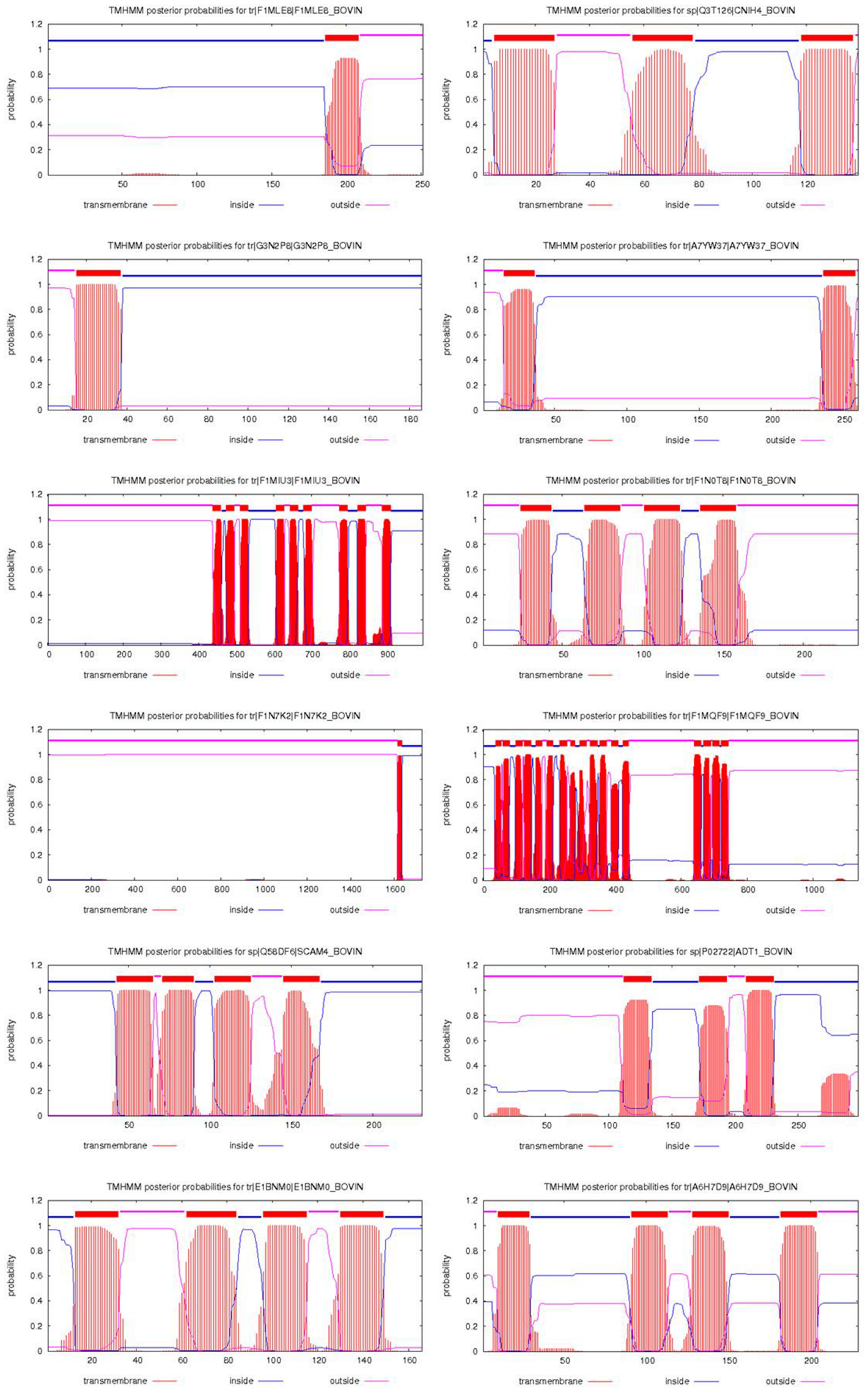


\section{Supplemental Figure 4-4}
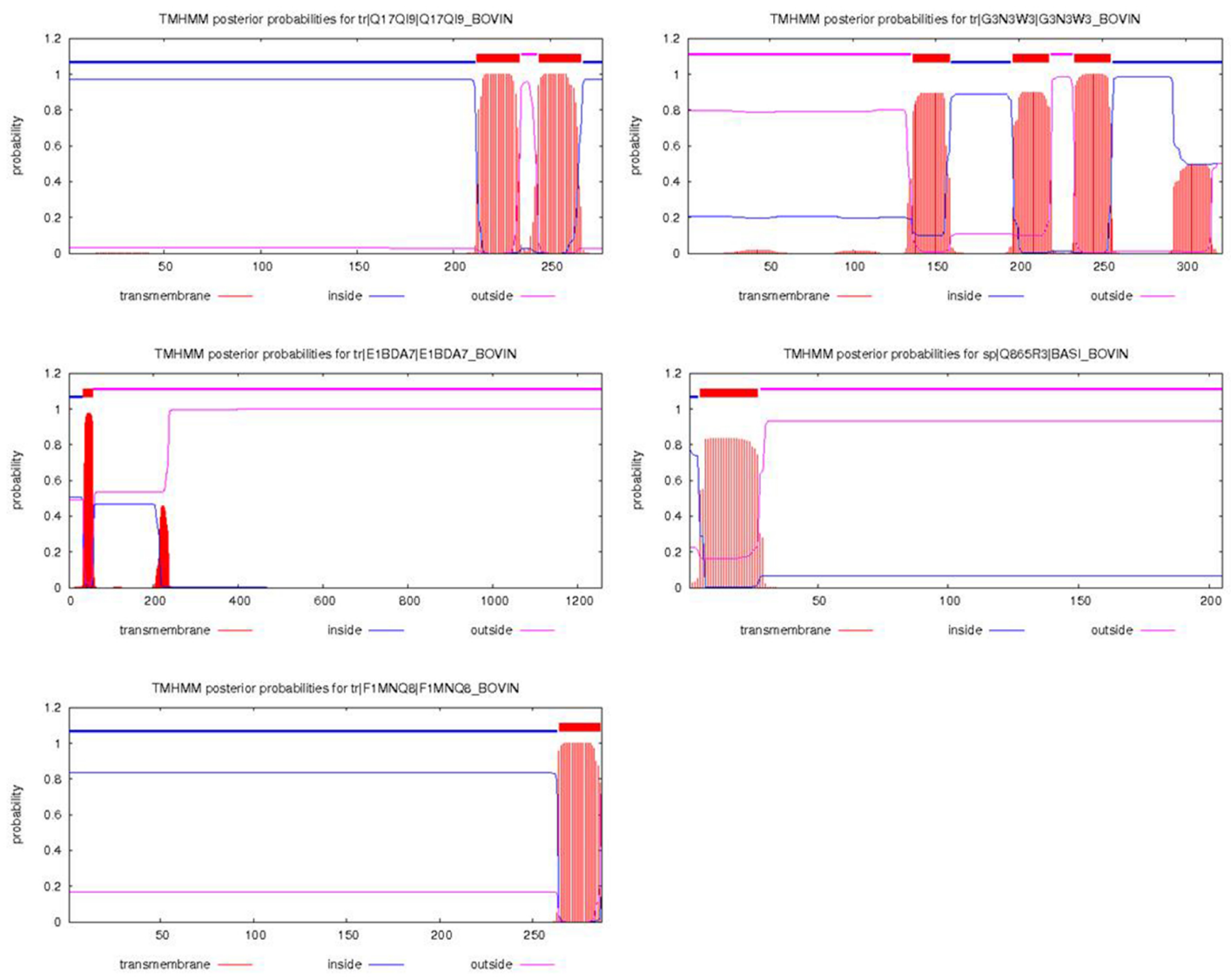


\section{Supplemental Figure 5}

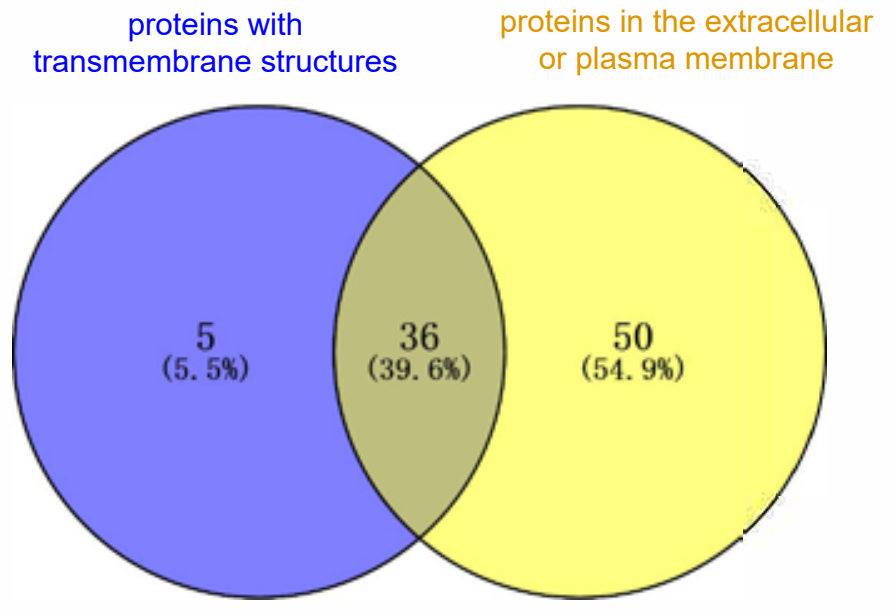




\section{Supplementary Figure 6}
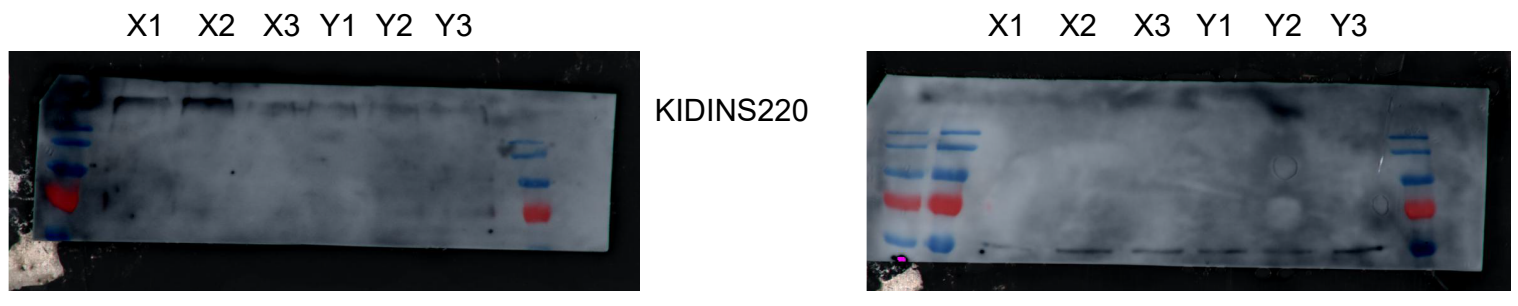

DPY19L2

$\begin{array}{llllll}X 1 & X 2 & X 3 & Y 1 & Y 2 & Y 3\end{array}$

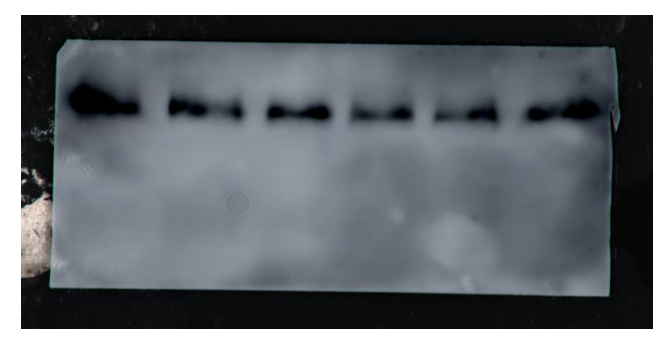

STX2

$\begin{array}{llllll}X 1 & X 2 & X 3 & Y 1 & Y 2 & Y 3\end{array}$

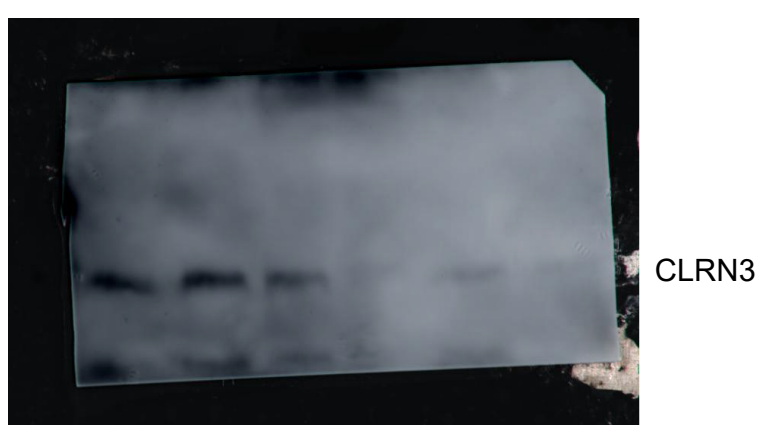

\section{$\begin{array}{llllll}X 1 & X 2 & X 3 & Y 1 & Y 2 & Y 3\end{array}$}

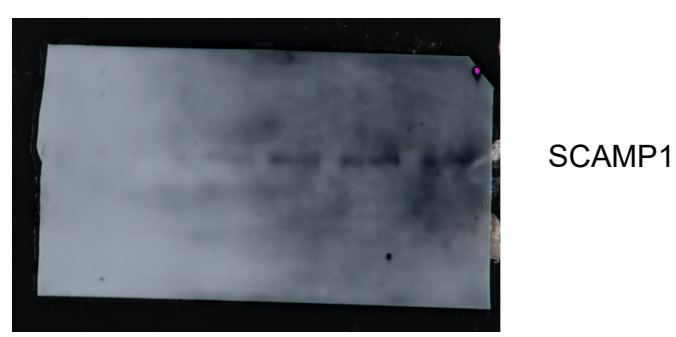

$\begin{array}{llllll}X 1 & X 2 & X 3 & Y 1 & Y 2 & Y 3\end{array}$

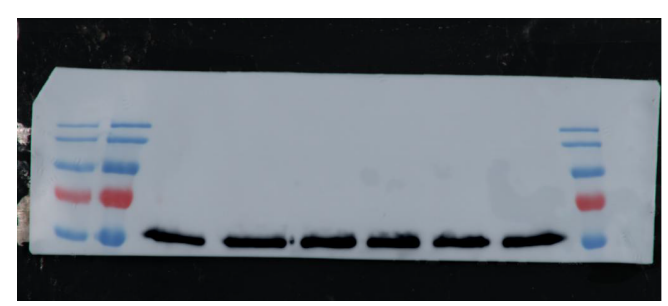




\section{Supplementary Figure 7}

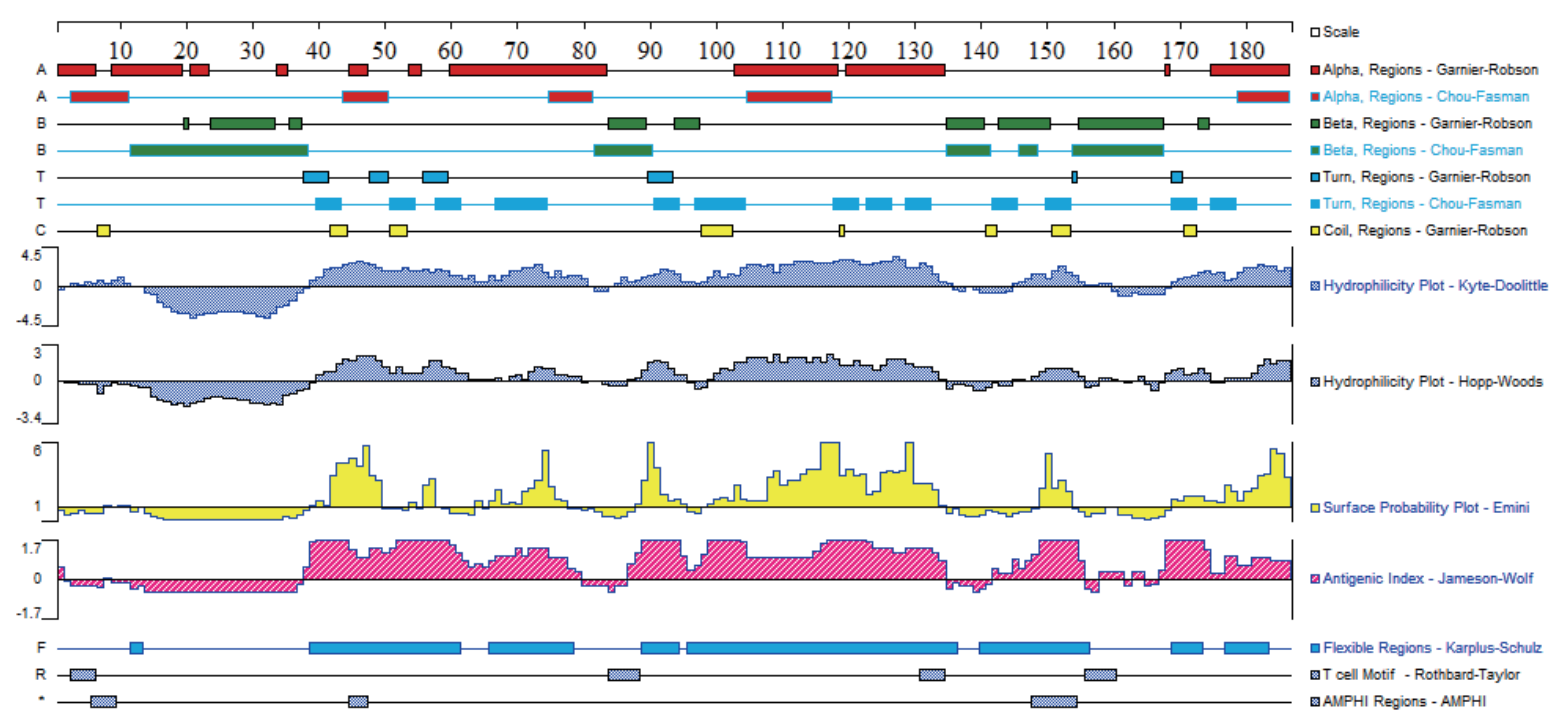

